\title{
Negative Thermal Expansion in the Plateau State of a Magnetically Frustrated Spinel
}

\author{
L. Rossi, ${ }^{1,2}$ A. Bobel, ${ }^{1,2}$ S. Wiedmann, ${ }^{1,2}$ R. Küchler, ${ }^{3}$ Y. Motome,${ }^{4}$ K. Penc,${ }^{5}$ N. Shannon, ${ }^{6,7}$ \\ H. Ueda, ${ }^{8}$ and B. Bryant ${ }^{1,2}$ \\ ${ }^{1}$ High Field Magnet Laboratory (HFML-EMFL), Radboud University, 6525 ED Nijmegen, Netherlands \\ ${ }^{2}$ Institute of Molecules and Materials, Radboud University, 6525 AJ Nijmegen, Netherlands \\ ${ }^{3}$ Max Planck Institute for Chemical Physics of Solids, Nöthnitzer Strasse 40, 01187 Dresden, Germany \\ ${ }^{4}$ Department of Applied Physics, The University of Tokyo, Hongo 7-3-1, Bunkyo-ku, Tokyo 113-8656, Japan \\ ${ }^{5}$ Institute for Solid State Physics and Optics, Wigner Research Centre for Physics, Hungarian Academy of Sciences, \\ H-1525 Budapest, Hungary \\ ${ }^{6}$ Okinawa Institute of Science and Technology Graduate University, Onna-son, Okinawa 904-0495, Japan \\ ${ }^{7}$ Department of Physics, Technische Universität München, D-85748 Garching, Germany \\ ${ }^{8}$ Department of Chemistry, Graduate School of Science, Kyoto University, Kyoto 606-8502, Japan
}

(Received 14 December 2018; revised manuscript received 4 April 2019; published 10 July 2019)

\begin{abstract}
We report on negative thermal expansion (NTE) in the high-field, half-magnetization plateau phase of the frustrated magnetic insulator $\mathrm{CdCr}_{2} \mathrm{O}_{4}$. Using dilatometry, we precisely map the phase diagram at fields of up to $30 \mathrm{~T}$ and identify a strong NTE associated with the collinear half-magnetization plateau for $B>27 \mathrm{~T}$. The resulting phase diagram is compared with a microscopic theory for spin-lattice coupling, and the origin of the NTE is identified as a large negative change in magnetization with temperature, coming from a nearly localized band of spin excitations in the plateau phase. These results provide useful guidelines for the discovery of new NTE materials.
\end{abstract}

DOI: 10.1103/PhysRevLett.123.027205

Frustrated magnets are materials with competing spin interactions, which cannot be simultaneously satisfied. While these materials are most famous as a playground for novel phases, such as quantum spin liquids [1,2], they also exhibit other, more technologically relevant properties, such as multiferroicity [3-5], and an enhanced magnetocaloric effect [6-10]. Negative thermal expansion (NTE) is another unusual phenomenon often observed in frustrated magnets [11-14]. This effect provides a route for the control of thermal expansion necessary to ensure the performance of high-precision devices [15], so theoretical models that can act as a guide for discovery of new NTE materials are highly valuable.

In frustrated magnets with a strong coupling between the spin and lattice degrees of freedom, the interplay between magnetic field and spin-lattice coupling produces a range of phases in which frustration is partially relieved, an effect known as "order by distortion" [16-21]. A paradigm for this type of behavior is provided by $\mathrm{Cr}$ spinels, which exhibit many different magnetically ordered phases as a function of the magnetic field [22-28]. Many of these systems exhibit NTE $[13,29,30]$, including the spinel $\mathrm{CdCr}_{2} \mathrm{O}_{4}$ in zero magnetic field [31]. This suggests that the unusual thermodynamic behavior may have a common origin; however, to date there is no general understanding of this phenomenon or how it is linked to spin-lattice coupling. Moreover, to obtain a complete picture of NTE in spinels, high-precision measurements are also needed for the ordered phases induced by the magnetic field.

In this Letter, we report on thermal expansion and magnetostriction measurements of the frustrated spinel $\mathrm{CdCr}_{2} \mathrm{O}_{4}$, in magnetic fields up to $30 \mathrm{~T}$. We map the phase diagram, which we compare to that derived from a microscopic model of spin-lattice coupling. The high-field, half-magnetization plateau phase exhibits enhanced thermal stability compared to theory, characteristic of a strong spin-lattice coupling in this phase. This state also shows a marked NTE, distinct from that observed in zero field. Starting from the same model of spin-lattice coupling, we develop a microscopic theory of this NTE and identify its origin as being a band of nearly localized magnetic excitations. These results provide a general framework for modeling and predicting NTE in pyrochlore lattices and in frustrated magnets in general.

The pyrochlore lattice, which consists of corner sharing tetrahedra, is a well-known stage for strong geometric frustration [32]. This structure is realized in the position of the $\mathrm{Cr}^{3+}$ ions in the chromium spinels $\mathrm{ACr}_{2} \mathrm{X}_{4}$, where $A$ is $\mathrm{Zn}, \mathrm{Cd}$, or $\mathrm{Hg}$ and $X$ is $\mathrm{O}, \mathrm{S}$, or Se. The strength and sign of the $\mathrm{Cr}-\mathrm{Cr}$ spin coupling depends strongly on the interatomic distance [33,34], leading to a strong coupling between spin ordering and lattice distortions. The oxide spinels $A \mathrm{Cr}_{2} \mathrm{O}_{4}$ all have antiferromagnetic spin coupling and are magnetically frustrated: because of the frustration they remain paramagnetic down to temperatures well below the Curie-Weiss temperature $\Theta_{\mathrm{CW}}$. At $T_{N}$ the spin 
frustration is relieved due to a spontaneous lattice distortion $[16,17]$, which allows a noncollinear spin-spiral antiferromagnetic ground state [35,36].

The $\mathrm{Cr}$ oxide spinels show another magnetostructural transition at high magnetic field, into a collinear state with one-half of the saturation magnetization, in which three of the spins in each tetrahedron point "up" and one points "down" $[18,21,22,37,38]$. This state has a constant magnetization across a wide range of magnetic fields, and it is thus often referred to as the "plateau" state. Both the magnetostructural transition at $T_{N}$ and the transition to the half-magnetization plateau are manifestations of the strong spin-lattice coupling in the $\mathrm{Cr}$ oxide spinels: a developed microscopic magnetoelastic theory $[18,20]$ describes how the plateau state is stabilized by the spin-lattice coupling [26,28].

In order to probe the interplay of frustration and spinlattice coupling, we performed thermal expansion and magnetostriction measurements of $\mathrm{CdCr}_{2} \mathrm{O}_{4}$ using capacitive dilatometry at low temperatures and high magnetic fields up to $30 \mathrm{~T}[39,40]$. This compound was chosen since it is highly frustrated, with $f=\left|\Theta_{\mathrm{CW}}\right| / T_{N} \approx 10$, highquality single crystals are available, and it is possible to reach the plateau phase in static (dc) high-field facilities. So far, zero-field thermal expansion measurements [31] and pulsed-field magnetostriction measurements [37] have been reported. We measured the strain $\Delta L / L$ along the [111] direction: the magnetic field is parallel to the [111] direction. The sample is clamped between two plates in the dilatometer, thus applying a small [111] uniaxial pressure. The effect of varying the applied pressure is discussed in the Supplemental Material [41]. We studied a series of single-crystal samples, all of which are platelike with wide (111) faces, around 3-5 $\mathrm{mm}$ in diameter and between 80 and $500 \mu \mathrm{m}$ thick.

High-field measurements were carried out with the sample mounted in a compact capacitive dilatometer in a $30 \mathrm{~T}$ resistive Bitter magnet [39]. Figure 1(a) presents thermal expansion from 4.2 to $10.4 \mathrm{~K}$ at zero field and at field increments up to $30 \mathrm{~T}$. Clearly visible up to $27 \mathrm{~T}$ is the magnetostructural transition at $T_{N}$, seen here on warming from the tetragonal antiferromagnetic phase to the cubic paramagnetic phase. $T_{N}$ decreases from $7.5 \mathrm{~K}$ at zero field to $5.5 \mathrm{~K}$ at $27 \mathrm{~T}$, while the measured [111] strain at $T_{N}$ remains constant. Above $26.5 \mathrm{~T}$, the transition from the high-temperature paramagnetic phase to the low-temperature half-magnetization plateau phase can be seen in the $\Delta L / L$ data as a peak superposed on a step [Fig. 1(b)]. The appearance or the absence of the peak is sample dependent, while the step was present in all the measured samples. Both phases are cubic - the paramagnetic state $F d \overline{3} m$ and the plateau state $\mathrm{P}_{3} 32$ [36] — so, from measurements on three samples, we can estimate a change in unit cell volume on cooling of $\Delta V / V \approx 2.2 \pm 0.9 \times 10^{-4}$ [44]. We can explain this increase in volume qualitatively as part of the general principle of the magnetoelastic theory that increased magnetization leads to increased unit cell volume, if

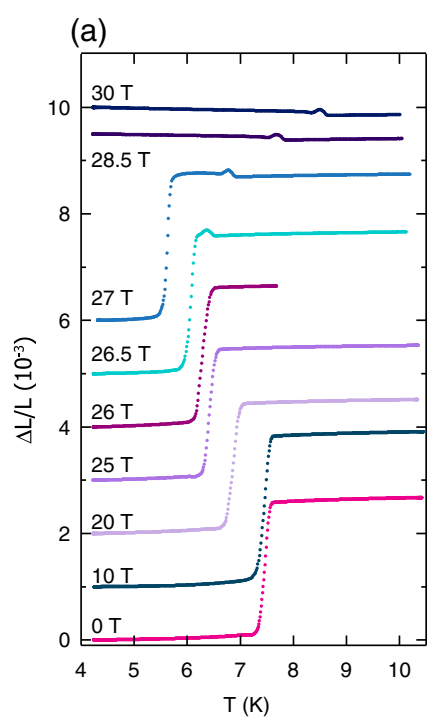

(b)
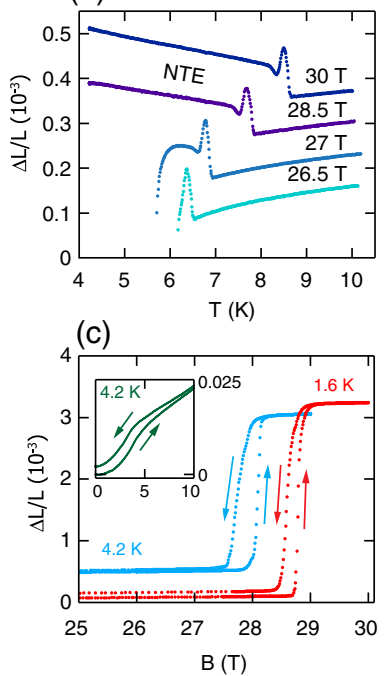

FIG. 1. Thermal expansion and magnetostriction measurements of $\mathrm{CdCr}_{2} \mathrm{O}_{4}$ at magnetic fields up to $30 \mathrm{~T}$, showing NTE for fields $B>27 \mathrm{~T}$. These results were used to determine the phase diagram shown in Fig. 2. (a) Thermal expansion measurements from 4.2 to $10.4 \mathrm{~K}$ in fields up to $30 \mathrm{~T}$, measured on warming, showing the variation of $T_{N}$ with field. (b) Detail of data in (a), for fields from 26.5 to $30 \mathrm{~T}$, showing transition from the paramagnetic state to the half-magnetization plateau state and the presence of NTE in the plateau state above 27 T. (a),(b) Curves have been offset for clarity. (c) Magnetostriction measurements from 0 to $30 \mathrm{~T}$ at 4.2 and $1.6 \mathrm{~K}$, showing a hysteretic transition from the antiferromagnetic state to the plateau state. (Inset) Magnetostriction up to $10 \mathrm{~T}$ at $4.2 \mathrm{~K}$, showing a hysteretic lowfield transition at around 4.5 T.

antiferromagnetic interactions are assumed [18]. Below this transition and above $27 \mathrm{~T}$, NTE is seen in the plateau phase, shown in Fig. 1(b).

In addition to thermal expansion, constant-temperature magnetostriction measurements were made, with field sweeps up to $30 \mathrm{~T}$, at temperatures between 1.3 and $4.2 \mathrm{~K}$. Figure 1(c) shows the results from 25 to $30 \mathrm{~T}$. We see a hysteretic transition from the tetragonal antiferromagnetic phase to the plateau phase, which is consistent with a first-order phase transition. The sweep rate close to the transition was $0.5 \mathrm{~T} / \mathrm{min}$. Previous pulsedfield measurements reported a colossal negative magnetostriction at the transition to the half-magnetization plateau, for both [111] and [110] directions [37,38]. In our [111] measurements we find a positive magnetostriction at this transition [45]. This is consistent both with measurements on $\mathrm{HgCr}_{2} \mathrm{O}_{4}$ [46] and with the magnetoelastic theory [18], in which jumps in magnetization are mirrored by unit cell expansion. Both the transition with field to the plateau phase [Fig. 1(c)] and the thermal transition at $T_{N}$ to the cubic, paramagnetic phase [Fig. 1(a)] have the same sign and similar magnitude in $\Delta L / L$. This indicates that these phases have a similar unit cell and supports the finding that the plateau phase also has overall cubic symmetry $[36,47]$. 
We also performed a second magnetostriction experiment in a superconductor magnet, between 0 and $15 \mathrm{~T}$ and from 2.2 to $7 \mathrm{~K}$. The inset in Fig. 1(c) presents magnetostriction data at $4.2 \mathrm{~K}$, which show a hysteretic low-field transition at around 4.5 T. A similar transition has previously been observed in magnetization data $[35,48]$. Based on ESR and optical spectroscopy measurements $[48,49]$, this has been interpreted as a transition from a helical structure to a commensurate canted spin structure. Neutron diffraction experiments, though, appear to rule out an incommensurate to commensurate transition [35], instead implying a rearrangement of spin-spiral domains between 2.5 and $6 \mathrm{~T}$. When the field is in the $a-c$ plane, in which the spins rotate in the spiral, a spin-flop is observed: since we apply the field along the [111] direction, we would expect a flop to a conical spin spiral.

We can summarize the results from the thermal expansion and magnetostriction measurements in a phase diagram, shown in Fig. 2(a). Three main phases are described: the high-temperature paramagnetic phase, the antiferromagnetic phase below $7.5 \mathrm{~K}$ and below $28.7 \mathrm{~T}$, and the high-field, half-magnetization plateau phase. Inside the antiferromagnetic phase, we identify a low-field transition, which increases from $4.3 \mathrm{~T}$ at $2.2 \mathrm{~K}$ to $5.1 \mathrm{~T}$ at $7 \mathrm{~K}$. Hysteresis is observed in all the transitions. We do not find any experimental evidence of the additional phase transition recently reported from sound velocity measurements [50], though the temperature dependence of the low-field transition is consistent with that report. Our new phase diagram is more precise for fields above $12 \mathrm{~T}$ than previous diagrams [24,37].

We can use a microscopic magnetoelastic theory to reproduce the experimental phase diagram and explain the presence of NTE in the plateau state. A simple Hamiltonian to account for the effects of spin-lattice coupling on the phase transitions in applied magnetic field in Cr spinels was introduced in [18],

$\mathcal{H}=\sum_{\langle i, j\rangle}\left(J\left(1-\alpha \rho_{i, j}\right) \mathbf{S}_{i} \cdot \mathbf{S}_{j}+\frac{K}{2} \rho_{i, j}^{2}\right)-\sum_{i} \mathbf{h} \cdot \mathbf{S}_{i}$,

where the summation is over the nearest-neighbor bonds on the pyrochlore lattice. $J$ is the antiferromagnetic exchange interaction, $\alpha$ is the spin-lattice coupling, $\rho_{i, j}$ is the change of the length of the bonds from the equilibrium distances in the paramagnetic phase, $K$ is the elastic constant, and $h$ is the applied magnetic field. In its simplest form, this theory reduces to solving an effective spin model with only two adjustable parameters,

$$
\mathcal{H}_{\mathrm{eff}}=J \sum_{\langle i j\rangle}\left(\mathbf{S}_{i} \cdot \mathbf{S}_{j}-b\left(\mathbf{S}_{i} \cdot \mathbf{S}_{j}\right)^{2}\right)-h \sum_{i} S_{i}^{z}
$$
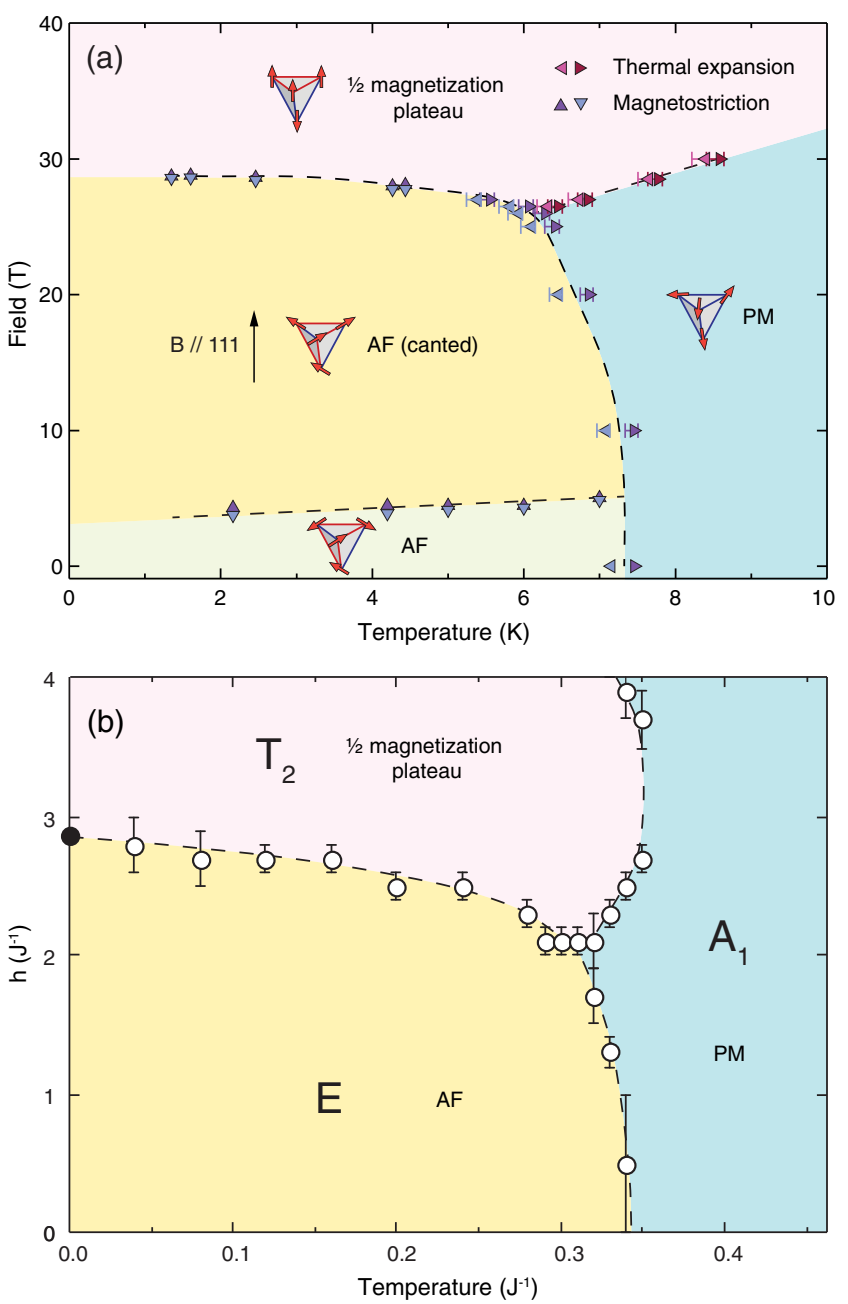

FIG. 2. Low-temperature, high magnetic field phase diagram for $\mathrm{CdCr}_{2} \mathrm{O}_{4}$. (a) Experimental phase diagram derived from magnetostriction and thermal expansion measurements, showing the antiferromagnetic (AF) and canted phases, half-magnetization plateau, and paramagnetic (PM) phases. Hysteresis is shown by separate points for increasing and decreasing field or temperature. For each phase a schematic spin tetrahedron is shown, with shorter bonds in blue and longer in red. (b) Theoretical phase diagram from Monte Carlo calculations based on the magnetoelastic theory, with the spin-lattice coupling parameter $b=0.1$ : the dominant lattice distortions in each phase $\left(A_{1}, E\right.$, and $\left.T_{2}\right)$ are shown.

where $b=J \alpha^{2} / K$ reflects the strength of the spin-lattice coupling. In the case of $\mathrm{CdCr}_{2} \mathrm{O}_{4}$, measurements of magnetization lead to an estimate of $b \approx 0.1$ [51].

The effective spin model [Eq. (2)] can be solved using classical Monte Carlo calculations [19,38], leading to the phase diagram shown in Fig. 2(b). Here, calculations have been carried out for four-sublattice order, stabilized by an additional third-neighbor interaction $J_{3}=-0.05 J$ [19]. However, very similar results are obtained for 16-sublattice order [52]. The phase diagram in Fig. 2(b) has been calculated for $b=0.1$ : for purposes of comparison, the 
results have been scaled for the experimental values of $T_{N}$ and the critical field $H_{c 1}$.

The Monte Carlo results reproduce the experimental phases well, particularly the $B-T$ dependence of the transitions to the antiferromagnetic phase. The main discrepancy between theory and experimental data is seen in the transition from the paramagnetic phase to the plateau phase, which experimentally has a considerably lower slope in $B / T$. This indicates that the plateau phase is stable to a higher temperature than the antiferromagnetic phase, as observed experimentally for $\mathrm{HgCr}_{2} \mathrm{O}_{4}$ [22]. By contrast, the Monte Carlo phase diagram [Fig. 2(b)] predicts that the plateau phase is stable only up to a temperature similar to $T_{N}$. In a more general formulation of the magnetoelastic theory, the coefficient of spin-lattice coupling $b$ takes on different values in phases in which tetrahedra undergo distortions with different symmetry $[18,53]$. In the present case, this leads to three distinct parameters: $b_{A_{1}}$ (uniform changes in volume), $b_{E}$ (tetragonal distortions, found in the AF phase), and $b_{T_{2}}$ (trigonal distortions, found in the half-magnetization plateau). From detailed comparison of the magnetoelastic theory to magnetization and ESR data for $\mathrm{CdCr}_{2} \mathrm{O}_{4}$, Kimura et al. [51] obtain $b_{A_{1}}=0.05, b_{E}=0.1$, and $b_{T_{2}}=$ 0.14. The Monte Carlo calculations shown in Fig. 2(b) assume $b_{A_{1}}=b_{E}=b_{T_{2}}=0.1$, and so probably underestimate $b_{T_{2}}$, and hence the thermal stability of the plateau state, explaining the discrepancy seen between the experimental and theoretical results.

We now turn to the issue of the NTE in the plateau phase. Several spinel compounds show NTE at zero field, including $\mathrm{CdCr}_{2} \mathrm{~S}_{4}$ [30], $\mathrm{ZnCr}_{2} \mathrm{Se}_{4}$ [13,54], and $\mathrm{CdCr}_{2} \mathrm{O}_{4}$ [31]. In all of these cases, the onset of NTE on cooling is in the paramagnetic phase, above the magnetic ordering temperature. Zero-field NTE in $\mathrm{CdCr}_{2} \mathrm{O}_{4}$ occurs exclusively within the paramagnetic phase for $45<T<140 \mathrm{~K}$ [31]. This contrasts with the results in the field, presented in Fig. 1, in which there is an abrupt onset of NTE at the magnetic ordering temperature, and the NTE occurs only within the low-temperature ordered phase. This suggests that the NTE observed within the plateau phase of $\mathrm{CdCr}_{2} \mathrm{O}_{4}$ may have a qualitatively different origin from that observed in the paramagnetic phase in zero field.

NTE in pyrochlore lattices is often attributed to strong spin-lattice coupling [13,30,31], but a general microscopic theory is lacking. It is therefore interesting to explore the predictions of the microscopic model of spin-lattice coupling [Eq. (1)]. These calculations, which are developed in the Supplemental Material [41], naturally divide into two parts: (1) an analysis of the different symmetry channels in which the lattice can distort, each with its own associated form of magnetic order, and (2) a characterization of the spin excitations within each different ordered phase. We find that the dominant magnetic contribution to the thermal expansion comes from the dependence of the $A_{1}$ (volume) distortion on the magnetization, viz,

$$
\frac{\partial}{\partial T} \frac{\Delta L}{L}=\frac{J \alpha}{K r_{0}} \frac{8}{3} M \frac{\partial M}{\partial T},
$$

where $r_{0}$ is the equilibrium lattice spacing, and $\alpha$ and $K$ are magnetoelastic couplings defined through Eq. (1).

NTE will occur when the magnetic contribution, Eq. (3), is both negative and sufficiently large to overcome the usual thermal expansion of the lattice [55]. This criterion is easily met in the half-magnetization plateau of $\mathrm{CdCr}_{2} \mathrm{O}_{4}$, where $\alpha$ and $M$ are individually large and positive, and the existence of a nearly localized band of spin excitations at low energies provides a microscopic explanation for the rapid decrease of magnetization with temperature $\partial M / \partial T<0$ [41].

This mechanism finds validation in both experiment [37], where the magnetization is observed to be sharply suppressed by increasing temperature, and in Monte Carlo simulation, as shown in Figs. S2 and S3 [41] and in Ref. [19]. It is also interesting to note that the NTE must be accompanied by a substantially enhanced magnetocaloric effect (MCE)

$$
\Gamma_{\mathrm{MCE}}=\left.\frac{\partial T}{\partial H}\right|_{S}=-\left.\frac{T}{C_{H}} \frac{\partial M}{\partial T}\right|_{H},
$$

coming from the same nearly localized band of excitations $[6,9,10]$. To the best of our knowledge, this has yet to be measured in experiment.

In making this analysis, we have assumed in Eq. (3) that $J$ does not vary with temperature: this is a good approximation for changes occurring within a given phase, although clearly $J$ can change substantially between phases with different lattice symmetry [51]. We can estimate the fractional change in $J$ with temperature within the plateau phase, from the known dependence of $T_{\mathrm{CW}}$ on the $\mathrm{Cr}-\mathrm{Cr}$ spacing [34] and the magnitude of the NTE, at $\Delta J / J \approx$ $1 \times 10^{-3} \mathrm{~K}^{-1}$. We note that $\mathrm{ZnCr}_{2} \mathrm{Se}_{4}$ shows a positive Curie-Weiss temperature while ordering antiferromagnetically, and this has been taken to imply that $J$ varies strongly with temperature [52]. However, we conclude that this attribute is not necessary to achieve NTE.

While Eq. (3) has been derived here in the context of the half-magnetization plateau of a $\mathrm{Cr}$ spinel, it has a much wider validity, and we would expect NTE to occur in many pyrochlore compounds where the above criteria are met: this is supported by measurements on other $\mathrm{Cr}$ spinels. In $\mathrm{CdCr}_{2} \mathrm{~S}_{4}$, NTE is observed to set in below $98 \mathrm{~K}$ in the paramagnetic phase and persists into the ferromagnetic phase [30]: here $\partial M / \partial T<0$ in both phases. $\mathrm{ZnCr}_{2} \mathrm{Se}_{4}$ shows NTE below $75 \mathrm{~K}$, but it is suppressed below $T_{N}=$ $21 \mathrm{~K}[13,54]$, where $\partial M / \partial T>0$. We would also predict NTE to occur in the high-field saturated magnetization phase of the oxide spinels [41].

In summary, we made thermal expansion and magnetostriction measurements of the frustrated spinel $\mathrm{CdCr}_{2} \mathrm{O}_{4}$, at low temperatures and at magnetic fields up to $30 \mathrm{~T}$. The experimental phase diagram strongly resembles that 
produced from Monte Carlo simulations of a minimal model of spin-lattice coupling, but diverges in that the plateau phase is more thermally stable than predicted, providing independent verification of the particularly strong spin-lattice coupling in this phase. We also observe NTE in the half-magnetization plateau phase and show how this can be explained in terms of the same microscopic model. We find the origin of the NTE to be a large, negative temperature derivative of magnetization, which comes from a band of nearly localized spin excitations.

These results are applicable across a broad range of spinel and pyrochlore magnets and potentially other frustrated magnets. They offer a route to the identification of other new NTE materials by suggesting that NTE is likely to occur in frustrated magnets where there is a collinear magnetic phase with a flat band. The results also imply a strong link between NTE and an enhanced magnetocaloric effect.

This work was supported by the Dutch funding organization Nederlandse Organisatie voor Wetenschappelijk Onderzoek (NWO-I) and by High Field Magnet Laboratory (HFML)-Radboud University (RU)/FOM, a member of the European Magnetic Field Laboratory (EMFL). R. K. is supported by the German Science Foundation through Project No. KU 3287/1-1, K. P. by Hungarian NKFIH Grant No. K 124176 and BMENanonotechnology and Materials Science FIKP Grant of EMMI (BME FIKP-NAT), and N.S. by the Theory of Quantum Matter Unit of the Okinawa Institute of Science and Technology Graduate University. K. P. acknowledges the hospitality of the Theory Quantum Matter Unit, OIST, where part of this work was carried out.

[1] P. A. Lee, Science 321, 1306 (2008).

[2] L. Savary and L. Balents, Rep. Prog. Phys. 80, 016502 (2017).

[3] S.-W. Cheong and M. Mostovoy, Nat. Mater. 6, 13 (2007).

[4] Y. Tokura, S. Seki, and N. Nagaosa, Rep. Prog. Phys. 77, 076501 (2014).

[5] M. Fiebig, T. Lottermoser, D. Meier, and M. Trassin, Nat. Rev. Mater. 1, 16046 (2016).

[6] M. E. Zhitomirsky, Phys. Rev. B 67, 104421 (2003).

[7] M. E. Zhitomirsky and A. Honecker, J. Stat. Mech. (2004) P07012.

[8] M. E. Zhitomirsky and H. Tsunetsugu, Prog. Theor. Phys. Suppl. 160, 361 (2005).

[9] O. Derzhko and J. Richter, Eur. Phys. J. B 52, 23 (2006).

[10] B. Schmidt, P. Thalmeier, and N. Shannon, Phys. Rev. B 76, 125113 (2007).

[11] A. Ramirez, C. Broholm, R. Cava, and G. Kowach, Physica (Amsterdam) 280B, 290 (2000).

[12] M. Shiga, K. Fujisawa, and H. Wada, J. Phys. Soc. Jpn. 62, 1329 (1993).

[13] J. Hemberger, H.-A. Krug von Nidda, V. Tsurkan, and A. Loidl, Phys. Rev. Lett. 98, 147203 (2007).
[14] B. Li, X. H. Luo, H. Wang, W. J. Ren, S. Yano, C.-W. Wang, J. S. Gardner, K.-D. Liss, P. Miao, S.-H. Lee, T. Kamiyama, R. Q. Wu, Y. Kawakita, and Z. D. Zhang, Phys. Rev. B 93, 224405 (2016).

[15] K. Takenaka, Front. Chem. 6, 267 (2018).

[16] Y. Yamashita and K. Ueda, Phys. Rev. Lett. 85, 4960 (2000).

[17] O. Tchernyshyov, R. Moessner, and S. L. Sondhi, Phys. Rev. Lett. 88, 067203 (2002); Phys. Rev. B 66, 064403 (2002).

[18] K. Penc, N. Shannon, and H. Shiba, Phys. Rev. Lett. 93, 197203 (2004).

[19] Y. Motome, K. Penc, and N. Shannon, J. Magn. Magn. Mater. 300, 57 (2006).

[20] D. L. Bergman, R. Shindou, G. A. Fiete, and L. Balents, Phys. Rev. B 74, 134409 (2006).

[21] N. Shannon, K. Penc, and Y. Motome, Phys. Rev. B 81, 184409 (2010).

[22] H. Ueda, H. Mitamura, T. Goto, and Y. Ueda, Phys. Rev. B 73, 094415 (2006).

[23] T. Rudolf, C. Kant, F. Mayr, J. Hemberger, V. Tsurkan, and A. Loidl, New J. Phys. 9, 76 (2007).

[24] E. Kojima, A. Miyata, S. Miyabe, S. Takeyama, H. Ueda, and Y. Ueda, Phys. Rev. B 77, 212408 (2008).

[25] V. Tsurkan, S. Zherlitsyn, V. Felea, S. Yasin, Y. Skourski, J. Deisenhofer, H.-A. Krug von Nidda, P. Lemmens, J. Wosnitza, and A. Loidl, Phys. Rev. Lett. 106, 247202 (2011).

[26] A. Miyata, H. Ueda, Y. Ueda, Y. Motome, N. Shannon, K. Penc, and S. Takeyama, J. Phys. Soc. Jpn. 80, 074709 (2011).

[27] A. Miyata, H. Ueda, Y. Ueda, H. Sawabe, and S. Takeyama, Phys. Rev. Lett. 107, 207203 (2011).

[28] A. Miyata, H. Ueda, Y. Ueda, Y. Motome, N. Shannon, K. Penc, and S. Takeyama, J. Phys. Soc. Jpn. 81, 114701 (2012).

[29] F. Yokaichiya, A. Krimmel, V. Tsurkan, I. Margiolaki, P. Thompson, H. N. Bordallo, A. N. Buchsteiner, N. Stüßer, D. N. Argyriou, and A. Loidl, Phys. Rev. B 79, 064423 (2009).

[30] M. Tachibana, N. Taira, and H. Kawaji, Solid State Commun. 151, 1776 (2011).

[31] S. Kitani, M. Tachibana, N. Taira, and H. Kawaji, Phys. Rev. B 87, 064402 (2013).

[32] J. S. Gardner, M. J. P. Gingras, and J. E. Greedan, Rev. Mod. Phys. 82, 53 (2010).

[33] A. N. Yaresko, Phys. Rev. B 77, 115106 (2008).

[34] H. Ueda and Y. Ueda, Phys. Rev. B 77, 224411 (2008).

[35] M. Matsuda, M. Takeda, M. Nakamura, K. Kakurai, A. Oosawa, E. Lelievre-Berna, J.-H. Chung, H. Ueda, H. Takagi, and S.-H. Lee, Phys. Rev. B 75, 104415 (2007).

[36] M. Matsuda, K. Ohoyama, S. Yoshii, H. Nojiri, P. Frings, F. Duc, B. Vignolle, G. L. J. A. Rikken, L.-P. Regnault, S.-H. Lee, H. Ueda, and Y. Ueda, Phys. Rev. Lett. 104, 047201 (2010).

[37] H. Ueda, H. A. Katori, H. Mitamura, T. Goto, and H. Takagi, Phys. Rev. Lett. 94, 047202 (2005).

[38] N. Shannon, H. Ueda, Y. Motome, K. Penc, H. Shiba, and H. Takagi, J. Phys. Conf. Ser. 51, 31 (2006).

[39] R. Küchler, T. Bauer, M. Brando, and F. Steglich, Rev. Sci. Instrum. 83, 095102 (2012).

[40] R. Küchler, A. Wörl, P. Gegenwart, M. Berben, B. Bryant, and S. Wiedmann, Rev. Sci. Instrum. 88, 083903 (2017). 
[41] See Supplemental Material at http://link.aps.org/ supplemental/10.1103/PhysRevLett.123.027205 for additional data on the effect of varying applied stress, the derivation of Eq. (3), and discussion of the microscopic origin of $d M / d T$. Supplemental material includes Refs. [42,43].

[42] A. Chacon, A. Bauer, T. Adams, F. Rucker, G. Brandl, R. Georgii, M. Garst, and C. Pfleiderer, Phys. Rev. Lett. 115, 267202 (2015).

[43] J.-H. Chung, Y. S. Song, S. Park, H. Ueda, Y. Ueda, and S.-H. Lee, J. Korean Phys. Soc. 62, 1900 (2013).

[44] In this estimate we are assuming that both phases have cubic symmetry, thus neglecting any structural anisotropy induced by magnetic field. Such distortions may be regarded as second-order corrections [18].

[45] The negative magnetostriction in Ref. [37] might have been caused by an anomalous strain induced by thermal contraction of an adhesive used to mount the sample. H. Ueda (private communication).

[46] Y. Tanaka, Y. Narumi, N. Terada, K. Katsumata, H. Ueda, U. Staub, K. Kindo, T. Fukui, T. Yamamoto, R. Kammuri, M. Hagiwara, A. Kikkawa, Y. Ueda, H. Toyokawa, T. Ishikawa, and H. Kitamura, J. Phys. Soc. Jpn. 76, 043708 (2007).
[47] T. Inami, K. Ohwada, M. Tsubota, Y. Murata, Y. H. Matsuda, H. Nojiri, H. Ueda, and Y. Murakami, J. Phys. Conf. Ser. 51, 502 (2006).

[48] S. Kimura, M. Hagiwara, H. Ueda, Y. Narumi, K. Kindo, H. Yashiro, T. Kashiwagi, and H. Takagi, Phys. Rev. Lett. 97, 257202 (2006).

[49] Y. Sawada, S. Kimura, K. Watanabe, and H. Ueda, J. Phys. Conf. Ser. 568, 042028 (2014).

[50] S. Zherlitsyn, V. Tsurkan, A. A. Zvyagin, S. Yasin, S. Erfanifam, R. Beyer, M. Naumann, E. Green, J. Wosnitza, and A. Loidl, Phys. Rev. B 91, 060406(R) (2015).

[51] S. Kimura, Y. Sawada, Y. Narumi, K. Watanabe, M. Hagiwara, K. Kindo, and H. Ueda, Phys. Rev. B 92, 144410 (2015).

[52] Y. Motome, K. Penc, and N. Shannon (unpublished).

[53] K. Penc, N. Shannon, Y. Motome, and H. Shiba, J. Phys. Condens. Matter 19, 145267 (2007).

[54] X. L. Chen, Z. R. Yang, W. Tong, Z. H. Huang, L. Zhang, S. L. Zhang, W. H. Song, L. Pi, Y. P. Sun, M. L. Tian, and Y. H. Zhang, J. Appl. Phys. 115, 083916 (2014).

[55] G. Hausch, Phys. Status Solidi (a) 18, 735 (1973). 